\title{
Face numbers and the fundamental group
}

\author{
Satoshi Murai* \\ Department of Pure and Applied Mathematics \\ Graduate School of Information Science and Technology \\ Osaka University, Suita, Osaka 565-0871, Japan \\ s-murai@ist.osaka-u.ac.jp
}

\author{
Isabella Novik ${ }^{\dagger}$ \\ Department of Mathematics \\ University of Washington \\ Seattle, WA 98195-4350, USA \\ novik@math. washington. edu
}

September 17, 2018

\begin{abstract}
We resolve a conjecture of Kalai asserting that the $g_{2}$-number of any simplicial complex $\Delta$ that represents a connected normal pseudomanifold of dimension $d \geq 3$ is at least as large as $\left(\begin{array}{c}d+2 \\ 2\end{array}\right) m(\Delta)$, where $m(\Delta)$ denotes the minimum number of generators of the fundamental group of $\Delta$. Furthermore, we prove that a weaker bound, $h_{2}(\Delta) \geq\left(\begin{array}{c}d+1 \\ 2\end{array}\right) m(\Delta)$, applies to any $d$-dimensional pure simplicial poset $\Delta$ all of whose faces of co-dimension $\geq 2$ have connected links. This generalizes a result of Klee. Finally, for a pure relative simplicial poset $\Psi$ all of whose vertex links satisfy Serre's condition $\left(S_{r}\right)$, we establish lower bounds on $h_{1}(\Psi), \ldots, h_{r}(\Psi)$ in terms of the $\mu$-numbers introduced by Bagchi and Datta.
\end{abstract}

\section{Introduction}

Our starting point is the lower bound theorem of Barnette [4], Kalai [11], and Fogelsanger [10] asserting that in the class of all simplicial complexes representing connected normal pseudomanifolds without boundary of dimension $d \geq 2$ and with $n$ vertices, the boundary complex of a stacked $(d+1)$-dimensional polytope simultaneously minimizes all the face numbers. In the last decade or so, a lot of effort went into strengthening these bounds by taking into consideration the topology of the complex in question, see, for instance, [13, 14, 15, 17, 18, 22]. While the theorems proved and techniques developed significantly increased our understanding of how the Betti numbers of a topological space $M$ affect possible face numbers of triangulations of $M$, the relationship between the face numbers and other topological invariants, for instance, the fundamental group, remained virtually unknown. The main goal of this note is to use the $\mu$-numbers introduced by Bagchi and Datta [3] to establish bounds on the face numbers in terms of the minimum number of generators of the fundamental group.

Let $\Delta$ be a $(d-1)$-dimensional simplicial complex. We denote by $f_{i}(\Delta)$ the number of $i$ dimensional faces of $\Delta$ for $i=-1,0,1, \ldots, d-1$, with $f_{-1}(\Delta)=1$ accounting for the empty face. The $f$-vector of $\Delta$ is the vector $f(\Delta)=\left(f_{-1}(\Delta), f_{0}(\Delta), \ldots, f_{d-1}(\Delta)\right)$. The $h$-vector of $\Delta, h(\Delta)=\left(h_{0}(\Delta), h_{1}(\Delta), \ldots, h_{d}(\Delta)\right)$, is defined by $\sum_{i=0}^{d} h_{i}(\Delta) x^{d-i}=\sum_{j=0}^{d} f_{j-1}(\Delta)(x-1)^{d-j}$;

\footnotetext{
${ }^{*}$ Research is partially supported by JSPS KAKENHI $16 \mathrm{~K} 05102$.

${ }^{\dagger}$ Research is partially supported by NSF grant DMS-1361423
} 
equivalently, $h_{i}(\Delta)=\sum_{j=0}^{i}(-1)^{i-j}\left(\begin{array}{c}d-j \\ i-j\end{array}\right) f_{j-1}(\Delta)$ for $i=0,1, \ldots, d$. The consecutive differences, $h_{i}(\Delta)-h_{i-1}(\Delta)$ are known as the $g$-numbers. Specifically, we will be interested in $g_{2}:=h_{2}-h_{1}$. When $\Delta$ is connected, we denote by $\pi_{1}(\Delta)$ the fundamental group of (the geometric realization of) $\Delta$ and by $m(\Delta)$ the minimum number of generators of $\pi_{1}(\Delta)$.

Kalai [1] conjectured that any triangulation $\Delta$ of a connected closed manifold of dimension $d \geq 3$ satisfies

$$
g_{2}(\Delta) \geq\left(\begin{array}{c}
d+2 \\
2
\end{array}\right) b_{1}(\Delta)
$$

where $b_{1}(\Delta)$ is the first Betti number of $\Delta$ (computed with coefficients in a field). In fact, he conjectured that under the same assumptions the following stronger inequality holds

$$
g_{2}(\Delta) \geq\left(\begin{array}{c}
d+2 \\
2
\end{array}\right) m(\Delta) .
$$

The second inequality is stronger than the first one since, as follows from the Hurewicz theorem, $m(\Delta) \geq b_{1}(\Delta)$ for any $\Delta$. Moreover, $m(\Delta)$ can be (arbitrarily) larger than $b_{1}(\Delta)$ : there exist manifolds with a perfect fundamental group, and hence vanishing first homology.

Inequality (1.1) was established in [17] (for oriented manifolds) and in [13] (for all manifolds). One of the main results of this paper is the proof of the other inequality:

Theorem 1.1. Let $\Delta$ be a simplicial complex of dimension $d \geq 3$. Assume further that $\Delta$ is a connected normal pseudomanifold. Then

$$
g_{2}(\Delta) \geq\left(\begin{array}{c}
d+2 \\
2
\end{array}\right) m(\Delta) .
$$

Moreover, if $d \geq 4$, then $g_{2}(\Delta)=\left(\begin{array}{c}d+2 \\ 2\end{array}\right) m(\Delta)$ if and only if $\Delta$ is a stacked manifold.

Our proof of Theorem 1.1 is based on studying the $\mu$-numbers introduced by Bagchi and Datta [3], and, specifically, on the following result verified in [13] (see the proof of Theorem 5.3 there). We postpone the definition of the $\mu$-numbers as well as several other definitions until the next section, and for now merely mention that the $\mu$-numbers satisfy the Morse inequalities; in particular, $\mu_{1}-\mu_{0} \geq b_{1}-b_{0}$.

Theorem 1.2. Let $\Delta$ be a simplicial complex of dimension $d \geq 3$. Assume further that $\Delta$ is a connected normal pseudomanifold. Then

$$
g_{2}(\Delta) \geq\left(\begin{array}{c}
d+2 \\
2
\end{array}\right)\left(\mu_{1}(\Delta)-\mu_{0}(\Delta)+1\right) \geq\left(\begin{array}{c}
d+2 \\
2
\end{array}\right) b_{1}(\Delta)
$$

Moreover, if $d \geq 4$, then $g_{2}(\Delta)=\left(\begin{array}{c}d+2 \\ 2\end{array}\right)\left(\mu_{1}(\Delta)-\mu_{0}(\Delta)+1\right)$ if and only if $g_{2}(\Delta)=\left(\begin{array}{c}d+2 \\ 2\end{array}\right) b_{1}(\Delta)$, which, in turn, happens if and only if $\Delta$ is a stacked manifold.

To prove Theorem 1.1. we show that any connected simplicial complex (and, even more generally, simplicial poset) $\Delta$ satisfies

$$
\mu_{1}(\Delta)-\mu_{0}(\Delta)+1 \geq m(\Delta) .
$$

In other words, the $\mu$-numbers appear to provide much finer information about the complex than the Betti numbers do. This inequality also leads to the following generalization of a result of Klee [12]. (Klee proved the same statement but in the special case of balanced simplicial posets.) 
Theorem 1.3. Let $\Delta$ be a pure connected simplicial complex (or, more generally, a simplicial poset) of dimension $d \geq 2$. Assume further that all faces of $\Delta$ of dimension $\leq d-2$ have connected links. Then

$$
h_{2}(\Delta) \geq\left(\begin{array}{c}
d+1 \\
2
\end{array}\right) m(\Delta) .
$$

In order to prove Theorem 1.3, we, in fact, establish the following more general result:

Theorem 1.4. Let $r \leq d$ and let $(\Delta, \Gamma)$ be a pure relative simplicial complex (or, more generally, a pure relative simplicial poset) of dimension $d$. If for each vertex $v$ of $\Delta$, the link $(\operatorname{lk}(v, \Delta), \operatorname{lk}(v, \Gamma))$ satisfies Serre's condition $\left(S_{r}\right)$, then

$$
h_{i}(\Delta, \Gamma) \geq\left(\begin{array}{c}
d+1 \\
i
\end{array}\right)\left(\sum_{j=1}^{i}(-1)^{i-j} \mu_{j-1}(\Delta, \Gamma ; \mathbb{K})+(-1)^{i} f_{-1}(\Delta, \Gamma)\right) \quad \text { for all } i \leq r .
$$

In particular, if $(\Delta, \Gamma)$ is Buchsbaum then these inequalities hold for all $i \leq d$.

Theorem 1.3 is then an immediate consequence of Inequality (1.3) along with the $(r=2 \&$ $\Gamma=\emptyset)$-case of Theorem 1.4.

The rest of the paper is organized as follows. In Section 2, we review basic results and definitions pertaining to simplicial complexes, including the definition of their $\mu$-numbers. Section 3 is devoted to the proof of Theorem 1.1. In Section 4 we verify Theorems 1.3 and 1.4 for the

case of simplicial complexes. Then, in Section 5, we review the notion of simplicial posets and explain how to extend Theorems 1.3 and 1.4 to this generality.

\section{Preliminaries}

The goal of this section is to review some basics of simplicial complexes and their $\mu$-numbers.

\subsection{Simplicial complexes}

A simplicial complex $\Delta$ on $V$ is a collection of subsets of $V$ that is closed under inclusion. A relative simplicial complex $\Psi$ on $V$ is a collection of subsets of $V$ with the property that there exist simplicial complexes $\Delta \supseteq \Gamma$ such that $\Psi=\Delta \backslash \Gamma$. We identify such a pair of simplicial complexes $(\Delta, \Gamma)$ with the relative simplicial complex $\Delta \backslash \Gamma$. A simplicial complex $\Delta$ will also be identified with $(\Delta, \emptyset)$. The elements of $\Delta \backslash \Gamma$ are called faces of $(\Delta, \Gamma)$, the 1-element faces are called vertices, and the maximal faces (under inclusion) are called facets. The dimension of a face $F \in \Delta \backslash \Gamma$ is the cardinality of $F$ minus one and the dimension of $(\Delta, \Gamma)$ is the maximum dimension of its faces. A relative simplicial complex is said to be pure if all of its facets have the same dimension.

Let $(\Delta, \Gamma)$ be a $(d-1)$-dimensional relative simplicial complex. We let $f_{i}(\Delta, \Gamma)$ denote the number of $i$-dimensional faces of $(\Delta, \Gamma)$; in particular, $f_{-1}(\Delta, \Gamma)$ is 1 if $\emptyset \in \Delta \backslash \Gamma$ and 0 if $\emptyset \notin \Delta \backslash \Gamma$. The components of the $h$-vector $h(\Delta, \Gamma)=\left(h_{0}(\Delta, \Gamma), \ldots, h_{d}(\Delta, \Gamma)\right)$ of $(\Delta, \Gamma)$ are defined by

$$
h_{i}(\Delta, \Gamma)=\sum_{j=0}^{i}(-1)^{i-j}\left(\begin{array}{l}
d-j \\
i-j
\end{array}\right) f_{j-1}(\Delta, \Gamma) \quad \text { for } i=0,1, \ldots, d .
$$


We denote by $H_{i}(\Delta, \Gamma ; \mathbb{K})$ the $i$ th homology of the pair $(\Delta, \Gamma)$ computed with coefficients in a field $\mathbb{K}$, and by $b_{i}(\Delta, \Gamma ; \mathbb{K})$ the $\mathbb{K}$-dimension of $H_{i}(\Delta, \Gamma ; \mathbb{K})$. Similarly, $\tilde{H}_{i}(\Delta, \Gamma ; \mathbb{K})$ and $\tilde{b}_{i}(\Delta, \Gamma ; \mathbb{K})$ stand for the $i$ th reduced homology of $(\Delta, \Gamma)$ and the $\mathbb{K}$-dimension of $\tilde{H}_{i}(\Delta, \Gamma ; \mathbb{K})$, respectively.

A simplicial complex $\Delta$ on $V$ gives rise to several new simplicial complexes. The link of a face $F$ in $\Delta$ is

$$
\operatorname{lk}(F, \Delta)=\{G \in \Delta: F \cup G \in \Delta, F \cap G=\emptyset\} .
$$

(We also define $\operatorname{lk}(F, \Delta)=\emptyset$ if $F$ is not a face of $\Delta$.) The induced subcomplex of $\Delta$ on $W \subseteq V$ is

$$
\Delta_{W}=\{F \in \Delta: F \subseteq W\} .
$$

If $v$ is not an element of $V$, then the cone over $\Delta$ with apex $v$ is the simplicial complex

$$
v * \Delta=\Delta \cup\{\{v\} \cup F: F \in \Delta\} .
$$

A $d$-dimensional simplicial complex $\Delta$ is a normal pseudomanifold (without boundary) if (1) it is pure, (2) each $(d-1)$-face of $\Delta$ is contained in exactly two facets of $\Delta$, and (3) the link of each non-empty face of dimension at most $d-2$ is connected. In particular, a triangulation of any closed manifold is a normal pseudomanifold.

A $d$-dimensional stacked manifold is a simplicial complex obtained by starting with several disjoint boundary complexes of the $(d+1)$-dimensional simplex and repeatedly forming connected sums and/or handle additions. In particular, connected stacked manifolds are homeomorphic to spheres or to connected sums of sphere bundles over the circle. Thus, when $d \geq 3$, the first Betti number of a $d$-dimensional stacked manifold $\Delta$ (computed over any field) coincides with $m(\Delta)$. This is the only property of stacked manifolds that will be used in this paper.

\subsection{The $\mu$-numbers}

We adopt the following definition of the $\mu$-numbers (cf., [15, Section 4]). For a finite set $V$, let $\mathcal{S}_{V}$ be the collection of all linear orderings of the elements of $V$. If $(\Delta, \Gamma)$ is a relative simplicial complex on $V$ with $|V|=n$ and $\varsigma=\left(v_{1}, \ldots, v_{n}\right) \in \mathcal{S}_{V}$, define

$$
\mu_{i}^{\varsigma}(\Delta, \Gamma ; \mathbb{K})=\sum_{k=1}^{n} \tilde{b}_{i-1}\left(\operatorname{lk}\left(v_{k}, \Delta_{\left\{v_{1}, \ldots, v_{k}\right\}}\right), \operatorname{lk}\left(v_{k}, \Gamma_{\left\{v_{1}, \ldots, v_{k}\right\}}\right) ; \mathbb{K}\right) \quad \text { for } 0 \leq i \leq \operatorname{dim}(\Delta, \Gamma)
$$

and

$$
\mu_{i}(\Delta, \Gamma ; \mathbb{K})=\frac{1}{n !} \sum_{\varsigma \in \mathcal{S}_{V}} \mu_{i}^{\varsigma}(\Delta, \Gamma ; \mathbb{K}) \quad \text { for } 0 \leq i \leq \operatorname{dim}(\Delta, \Gamma) .
$$

When $i \leq 1$, we write $\mu_{i}^{\varsigma}(\Delta, \Gamma)$ instead of $\mu_{i}^{\varsigma}(\Delta, \Gamma ; \mathbb{K})$ and $\mu_{i}(\Delta, \Gamma)$ instead of $\mu_{i}(\Delta, \Gamma ; \mathbb{K})$ since these $\mu$-numbers do not depend on $\mathbb{K}$.

The $\mu^{\varsigma}$-numbers were essentially introduced by Brehm and Kühnel [6] while the $\mu$-numbers are due to Bagchi and Datta [3]. (Note that our $\mu_{0}$ is slightly different than $\mu_{0}$ of [3].) As can be seen from the above definition, these numbers are related to a version of the Morse theory for simplicial complexes (cf. [3, Remark 2.11]). In particular, they satisfy the following inequalities known as the Morse inequalities (see [15, Lemma 4.2]). 
Lemma 2.1. Let $(\Delta, \Gamma)$ be a relative simplicial complex on $V$ and let $\varsigma \in \mathcal{S}_{V}$. Then

$$
\sum_{j=0}^{i}(-1)^{i-j} b_{j}(\Delta, \Gamma ; \mathbb{K}) \leq \sum_{j=0}^{i}(-1)^{i-j} \mu_{j}^{\varsigma}(\Delta, \Gamma ; \mathbb{K}) \quad \text { for all } i \geq 0 .
$$

Note that since $\mu_{j}(\Delta, \Gamma ; \mathbb{K})$ is the average of $\mu_{j}^{\varsigma}(\Delta, \Gamma ; \mathbb{K})$ over all orderings $\varsigma \in \mathcal{S}_{V}$, the above inequalities continue to hold with the $\mu^{\varsigma}$-numbers replaced by the $\mu$-numbers.

\subsection{Stanley-Reisner rings and modules}

We now turn to reviewing the Stanley-Reisner modules and their connection to the $\mu$-numbers. Let $\Delta$ be a simplicial complex on $V$ and let $S=\mathbb{K}\left[x_{v}: v \in V\right]$ be the polynomial ring over a field $\mathbb{K}$ with $\operatorname{deg} x_{v}=1$ for all $v \in V$. The ideal

$$
I_{\Delta}=\left(x_{v_{1}} x_{v_{2}} \cdots x_{v_{k}}:\left\{v_{1}, v_{2}, \ldots, v_{k}\right\} \notin \Delta\right) \subseteq S
$$

is called the Stanley-Reisner ideal of $\Delta$ and the ring $\mathbb{K}[\Delta]=S / I_{\Delta}$ is called the StanleyReisner ring of $\Delta$. Similarly, if $(\Delta, \Gamma)$ is a relative simplicial complex, then the StanleyReisner module of $(\Delta, \Gamma)$ is the $S$-module

$$
\mathbb{K}[\Delta, \Gamma]=I_{\Gamma} / I_{\Delta}
$$

When $M$ is a finitely generated graded $S$-module, the numbers

$$
\beta_{i, j}(M)=\operatorname{dim}_{\mathbb{K}} \operatorname{Tor}_{i}^{S}(M, \mathbb{K})_{j}
$$

are called the graded Betti numbers of $M$. Here $N_{k}$ denotes the degree- $k$ graded component of a graded $S$-module $N$. For a relative simplicial complex $(\Delta, \Gamma)$ on $V$ with $|V|=n$, we define the $\tilde{\sigma}$-numbers of $(\Delta, \Gamma)$ by

$$
\tilde{\sigma}_{i-1}(\Delta, \Gamma ; \mathbb{K})=\frac{1}{n+1} \sum_{k=0}^{n} \frac{1}{\left(\begin{array}{l}
n \\
k
\end{array}\right)} \beta_{k-i, k}(\mathbb{K}[\Delta, \Gamma]) .
$$

We will rely on the following interpretation of the $\mu$-numbers in terms of the $\tilde{\sigma}$-numbers, see 15 , Sections 4 \& 6]. This interpretation is a consequence of Hochster's formula for the graded Betti numbers of Stanley-Reisner modules.

Lemma 2.2. Let $(\Delta, \Gamma)$ be a relative simplicial complex on $V$. Then

$$
\mu_{i}(\Delta, \Gamma ; \mathbb{K})=\sum_{v \in V} \tilde{\sigma}_{i-1}(\operatorname{lk}(v, \Delta), \operatorname{lk}(v, \Gamma) ; \mathbb{K}) \quad \text { for } i \geq 0 .
$$

\section{The $\mu$-numbers and the fundamental group}

In this section we prove Theorem 1.1, This will require a few lemmas.

Lemma 3.1. Let $\Delta$ and $\Gamma$ be simplicial complexes. Assume further that $\Delta$ is connected, $\Gamma$ is contractible, and $\Delta \cap \Gamma$ has exactly two connected components. Then

$$
m(\Delta \cup \Gamma) \leq m(\Delta)+1 .
$$


Proof: Denote the two connected components of $\Delta \cap \Gamma$ by $\Sigma_{1}$ and $\Sigma_{2}$. Since $\Delta$ is connected, there is a path

$$
\gamma=\left\{\left\{v_{1}, v_{2}\right\},\left\{v_{2}, v_{3}\right\}, \ldots,\left\{v_{s-1}, v_{s}\right\}\right\} \subset \Delta
$$

such that $v_{1} \in \Sigma_{1}, v_{s} \in \Sigma_{2}$, and $v_{2}, \ldots, v_{s-1}$ do not belong to $\Gamma$. Let $\Gamma^{\prime}=\Gamma \cup \gamma$. Since $\Gamma$ is contractible, $\Gamma^{\prime}$ is homotopy equivalent to $\mathbb{S}^{1}$. Furthermore, $\Delta \cap \Gamma^{\prime}=\Sigma_{1} \cup \Sigma_{2} \cup \gamma$ is connected. Therefore, by the Seifert-van Kampen theorem, the fundamental group $\pi_{1}\left(\Delta \cup \Gamma^{\prime}\right)$ is a quotient of the free product $\pi_{1}(\Delta) * \pi_{1}\left(\Gamma^{\prime}\right)=\pi_{1}(\Delta) * \mathbb{Z}$. Since $\Delta \cup \Gamma^{\prime}=\Delta \cup \Gamma$, this fact implies the desired inequality.

Lemma 3.2. Let $\Delta$ be a connected simplicial complex, let $\Gamma_{1}, \Gamma_{2}, \ldots, \Gamma_{s}$ be several connected and pairwise disjoint subcomplexes of $\Delta$, and let $v$ be an element that is not in $\Delta$. Then

$$
m\left(\Delta \cup\left(v *\left(\Gamma_{1} \cup \cdots \cup \Gamma_{s}\right)\right)\right) \leq m(\Delta)+s-1 .
$$

Proof: Assume first that $s \geq 2$. Since $v * \Gamma_{s}$ is contractible and since its intersection with $\Delta \cup\left(v *\left(\Gamma_{1} \cup \cdots \cup \Gamma_{s-1}\right)\right)$ consists of two connected components, namely, the vertex $v$ and the complex $\Gamma_{s}$, we obtain from Lemma 3.1 that

$$
m\left(\Delta \cup\left(v *\left(\Gamma_{1} \cup \cdots \cup \Gamma_{s}\right)\right)\right) \leq m\left(\Delta \cup\left(v *\left(\Gamma_{1} \cup \cdots \cup \Gamma_{s-1}\right)\right)\right)+1 .
$$

Thus, to complete the proof, it suffices to show that $m\left(\Delta \cup\left(v * \Gamma_{1}\right)\right) \leq m(\Delta)$. Indeed, since $\Delta \cap\left(v * \Gamma_{1}\right)=\Gamma_{1}$ is connected and $v * \Gamma_{1}$ is contractible, this inequality is a consequence of the Seifert-van Kampen theorem. The statement follows.

For the rest of this section, it is convenient to extend the definition of $m(\Delta)$ to the class of all simplicial complexes (including the disconnected ones) by letting $m(\Delta)$ be the sum of the $m$-numbers of the connected components of $\Delta$. With this definition in hand, we can state the following result that together with Theorem 1.2 immediately implies the inequality part of Theorem 1.1.

Theorem 3.3. Let $\Delta$ be a simplicial complex on $V$ and let $\varsigma=\left(v_{1}, \ldots, v_{n}\right) \in \mathcal{S}_{V}$. Then

$$
\mu_{1}^{\varsigma}(\Delta)-\mu_{0}^{\varsigma}(\Delta) \geq m(\Delta)-b_{0}(\Delta)
$$

In particular, if $\Delta$ is connected, then $\mu_{1}(\Delta)-\mu_{0}(\Delta)+1 \geq m(\Delta)$.

Proof: The "in particular" part follows from the first part since $\mu_{1}(\Delta)-\mu_{0}(\Delta)+1$ is the average of $\mu_{1}^{\varsigma}(\Delta)-\mu_{0}^{\varsigma}(\Delta)+1$ over all orderings $\varsigma$. To prove the first part, we use induction on $|V|=n$. The desired inequality does hold when $\Delta=\{\emptyset\}$ (with both sides equal to 0 ). Thus we may assume that $n \geq 1$.

Let $\varsigma^{\prime}=\left(v_{1}, \ldots, v_{n-1}\right)$ and $\Delta^{\prime}=\Delta_{\left\{v_{1}, \ldots, v_{n-1}\right\}}$. Then $\Delta=\Delta^{\prime} \cup\left(v_{n} * \operatorname{lk}\left(v_{n}, \Delta\right)\right)$ and

$$
\mu_{1}^{\varsigma}(\Delta)-\mu_{0}^{\varsigma}(\Delta)=\mu_{1}^{\varsigma^{\prime}}\left(\Delta^{\prime}\right)-\mu_{0}^{\varsigma^{\prime}}\left(\Delta^{\prime}\right)+\left(\tilde{b}_{0}\left(\operatorname{lk}\left(v_{n}, \Delta\right)\right)-\tilde{b}_{-1}\left(\operatorname{lk}\left(v_{n}, \Delta\right)\right)\right) .
$$

Therefore, by the induction hypothesis, to complete the proof, it suffices to show that

$$
m\left(\Delta^{\prime}\right)-b_{0}\left(\Delta^{\prime}\right)+\left(\tilde{b}_{0}\left(\operatorname{lk}\left(v_{n}, \Delta\right)\right)-\tilde{b}_{-1}\left(\operatorname{lk}\left(v_{n}, \Delta\right)\right)\right) \geq m(\Delta)-b_{0}(\Delta) .
$$


If $\operatorname{lk}\left(v_{n}, \Delta\right)=\{\emptyset\}$, then $\Delta$ is the disjoint union of $\Delta^{\prime}$ and the vertex $v_{n}$. Hence $m(\Delta)=m\left(\Delta^{\prime}\right)$ and $b_{0}(\Delta)=b_{0}\left(\Delta^{\prime}\right)+1$, while $\tilde{b}_{0}\left(\operatorname{lk}\left(v_{n}, \Delta\right)\right)-\tilde{b}_{-1}\left(\operatorname{lk}\left(v_{n}, \Delta\right)\right)=0-1=-1$, yielding that (3.1) holds as equality in this case.

Suppose $\operatorname{lk}\left(v_{n}, \Delta\right) \neq\{\emptyset\}$. Let $t=b_{0}\left(\Delta^{\prime}\right)$ and let $\Gamma_{1}, \ldots, \Gamma_{t}$ be the connected components of $\Delta^{\prime}$. Let $\Sigma_{i}=\Gamma_{i} \cap \operatorname{lk}\left(v_{n}, \Delta\right)$ for $i=1,2, \ldots, t$. Without loss of generality, assume that the number of connected components of $\Sigma_{i}$ is $s_{i} \geq 1$ for $i=1,2, \ldots, \ell$ and that $\Sigma_{i}$ is empty for $i>\ell$. Then

$$
\begin{aligned}
& \sum_{k=1}^{\ell} s_{i}=b_{0}\left(\operatorname{lk}\left(v_{n}, \Delta\right)\right)=\tilde{b}_{0}\left(\operatorname{lk}\left(v_{n}, \Delta\right)\right)-\tilde{b}_{-1}\left(\operatorname{lk}\left(v_{n}, \Delta\right)\right)+1, \\
& b_{0}\left(\Delta^{\prime}\right)-b_{0}(\Delta)=\ell-1, \\
& m\left(\Gamma_{i} \cup\left(v_{n} * \operatorname{lk}\left(v_{n}, \Delta\right)\right)\right) \leq m\left(\Gamma_{i}\right)+s_{i}-1 \quad \text { for } i=1,2, \ldots, \ell,
\end{aligned}
$$

where the last inequality follows from Lemma 3.2. Since

$$
\begin{aligned}
\Delta & =\Delta^{\prime} \cup\left(v_{n} * \operatorname{lk}\left(v_{n}, \Delta\right)\right) \\
& =\left(\Gamma_{1} \cup\left(v_{n} * \Sigma_{1}\right)\right) \cup \cdots \cup\left(\Gamma_{\ell} \cup\left(v_{n} * \Sigma_{\ell}\right)\right) \cup \Gamma_{\ell+1} \cup \cdots \cup \Gamma_{t},
\end{aligned}
$$

and since $\Gamma_{k} \cup\left(v_{n} * \Sigma_{k}\right)$ and $\Gamma_{k^{\prime}} \cup\left(v_{n} * \Sigma_{k^{\prime}}\right)$ intersect in the same single vertex $v_{n}$ for all $1 \leq k<k^{\prime} \leq \ell$, we infer from (3.2)-(3.4) (and the Seifert-van Kampen theorem) that

$$
\begin{aligned}
m(\Delta) & =m\left(\Gamma_{1} \cup\left(v_{n} * \Sigma_{1}\right)\right)+\cdots+m\left(\Gamma_{\ell} \cup\left(v_{n} * \Sigma_{\ell}\right)\right)+m\left(\Gamma_{\ell+1}\right)+\cdots+m\left(\Gamma_{t}\right) \\
& \leq \sum_{k=1}^{t} m\left(\Gamma_{k}\right)+\sum_{k=1}^{\ell}\left(s_{k}-1\right) \\
& =m\left(\Delta^{\prime}\right)+\left(\tilde{b}_{0}\left(\operatorname{lk}\left(v_{n}, \Delta\right)\right)-\tilde{b}_{-1}\left(\operatorname{lk}\left(v_{n}, \Delta\right)\right)\right)+\left(b_{0}(\Delta)-b_{0}\left(\Delta^{\prime}\right)\right),
\end{aligned}
$$

and Inequality (3.1) follows.

To complete the poof of Theorem [1.1, it only remains to discuss the case of equality. Since $g_{2}(\Delta) \geq\left(\begin{array}{c}d+2 \\ 2\end{array}\right)\left(\mu_{1}(\Delta)-\mu_{0}(\Delta)+1\right) \geq\left(\begin{array}{c}d+2 \\ 2\end{array}\right) m(\Delta)$, the statement follows easily from the equality case of Theorem 1.2 along with the observation that for a stacked manifold $\Delta$ of dimension $\geq 3$, $m(\Delta)=b_{1}(\Delta)$ (see the end of Section 2.1).

It is known that if $\Delta$ is a 3-dimensional connected normal pseudomanifold, then $g_{2}(\Delta)=$ $10 b_{1}(\Delta)$ if and only if $\Delta$ is a stacked manifold. However, the class of 3 -dimensional normal pseudomanifolds that satisfy $g_{2}(\Delta)=10\left(\mu_{1}(\Delta)-\mu_{0}(\Delta)+1\right)$ is larger: it consists of simplicial manifolds all of whose vertex links are stacked spheres, and, for instance, includes the boundary complexes of 4-dimensional cyclic polytopes. (See the proof of [13, Theorem 5.3] for both statements.) It would be interesting to understand which 3-dimensional connected normal pseudomanifolds satisfy the inequality $g_{2}(\Delta) \geq 10 \mathrm{~m}(\Delta)$ of Theorem 1.1 as equality.

It is also worth mentioning that the proof of Theorem 1.2 given in [13], in fact, shows that the inequality part of this theorem applies to a larger class of complexes; specifically, it holds for an arbitrary $d$-dimensional pure simplicial complex all of whose vertex links are generically $d$-rigid. As Buchsbaum* simplicial complexes (introduced in [2]) of dimension $d \geq 3$ have generically $d$-rigid vertex links (see [2, Theorem 4.1] for an even stronger result), we conclude that such complexes satisfy the inequality of Theorem 1.2 and hence also of Theorem 1.1;

Theorem 3.4. Let $\Delta$ be a connected simplicial complex of dimension $d \geq 3$. Assume further that $\Delta$ is Buchsbaum*. Then $g_{2}(\Delta) \geq\left(\begin{array}{c}d+2 \\ 2\end{array}\right) m(\Delta)$. 


\section{Lower bounds on the $h$-numbers}

In this section we consider pure simplicial complexes all of whose vertex links satisfy Serre's condition $\left(S_{r}\right)$; we establish lower bounds on the $h$-numbers of such complexes in terms of their $\mu$-numbers. A relative simplicial complex $(\Delta, \Gamma)$ satisfies Serre's condition $\left(S_{r}\right)$ (over $\left.\mathbb{K}\right)$ if for every face $F \in \Delta$ (including the empty face),

$$
\tilde{H}_{i}(\operatorname{lk}(F, \Delta), \operatorname{lk}(F, \Gamma) ; \mathbb{K})=0 \quad \text { for all } i<\min \{r-1, \operatorname{dim}(\operatorname{lk}(F, \Delta), \operatorname{lk}(F, \Gamma))\} .
$$

We recall some basic facts on complexes satisfying Serre's conditions. These conditions arise from Serre's $\left(S_{r}\right)$ condition in commutative algebra (see [ㅁ, Section 2.1] for the definition). It is known that if $r \geq 2$, then a finitely generated graded module $M$ over a polynomial ring satisfies Serre's condition $\left(S_{r}\right)$ if and only if its deficiency module $K_{M}^{j}$ (that is, the Matlis dual of the $j$ th local cohomology module of $M$ ) has Krull dimension $\leq j-r$ for all $0 \leq j<d$ (see [19, Lemma 3.2.1]). Consequently, for $r \geq 2$, it follows from Hochster's formula for local cohomology modules [1, Theorem 1.8] that a $(d-1)$-dimensional relative simplicial complex $(\Delta, \Gamma)$ satisfies $\left(S_{r}\right)$ if and only if $\mathbb{K}[\Delta, \Gamma]$ satisfies Serre's condition $\left(S_{r}\right)$. Thus, a $(d-1)$-dimensional relative complex $(\Delta, \Gamma)$ satisfies $\left(S_{d}\right)$ if and only if $(\Delta, \Gamma)$ is Cohen-Macaulay; furthermore, a $(d-1)$-dimensional simplicial complex $\Delta$ satisfies $\left(S_{2}\right)$ if and only if it is pure and for every face $F \in \Delta$ of dimension $\leq d-3$, the link of $F, \operatorname{lk}(F, \Delta)$, is connected.

The following result was proved in [16].

Theorem 4.1. Let $(\Delta, \Gamma)$ be a $(d-1)$-dimensional relative simplicial complex. If $(\Delta, \Gamma)$ satisfies $\left(S_{r}\right)$ over $\mathbb{K}$ then there is a sequence of linear forms $\theta_{1}, \ldots, \theta_{d}$ such that

(i) the multiplication map

$$
\times \theta_{i}:\left(\mathbb{K}[\Delta, \Gamma] /\left(\theta_{1}, \ldots, \theta_{i-1}\right) \mathbb{K}[\Delta, \Gamma]\right)_{k-1} \rightarrow\left(\mathbb{K}[\Delta, \Gamma] /\left(\theta_{1}, \ldots, \theta_{i-1}\right) \mathbb{K}[\Delta, \Gamma]\right)_{k}
$$

is injective for all $i=1,2, \ldots, d$ and all $k \leq r$, and

(ii) $\operatorname{dim}_{\mathbb{K}}\left(\mathbb{K}[\Delta, \Gamma] /\left(\theta_{1}, \ldots, \theta_{d}\right) \mathbb{K}[\Delta, \Gamma]\right)_{i}=h_{i}(\Delta, \Gamma)$ for all $i \leq r$.

While Theorem 4.1 was verified in [16] only for the case of Stanley-Reisner rings, it also holds in the generality of Stanley-Reisner modules. Indeed, the proofs given in [16] apply to an arbitrary squarefree module (a notion introduced and studied by Yanagawa in [23]), while by [23. Lemma 2.3] all Stanley-Reisner modules are squarefree. (See also the proof of [24, Theorem 5.6].)

Since the proof of the main result of this section follows the same outline as the proof of [15, Theorem 6.5], instead of providing complete details we only sketch the main ideas. The following result is [15, Lemma 5.5].

Lemma 4.2. Let $S=\mathbb{K}\left[x_{1}, \ldots, x_{n}\right]$, let $M$ be a finitely generated graded $S$-module, and let $\theta_{1}, \ldots, \theta_{d}$ be linear forms. If

$$
\times \theta_{i}:\left(M /\left(\theta_{1}, \ldots, \theta_{i-1}\right) M\right)_{k-1} \rightarrow\left(M /\left(\theta_{1}, \ldots, \theta_{i-1}\right) M\right)_{k}
$$

is injective for all $i=1,2, \ldots, d$ and all $k \leq r$, then

$$
\sum_{k \geq 0}(-1)^{k} \beta_{i+k, i+\ell}(M) \leq \sum_{k \geq 0}(-1)^{k}\left(\begin{array}{c}
n-d \\
i+k
\end{array}\right) \operatorname{dim}_{\mathbb{K}}\left(M /\left(\theta_{1}, \ldots, \theta_{d}\right) M\right)_{\ell-k}
$$

for all $i \geq 0$ and $\ell \leq r-1$. 
Theorem 4.1 asserts that if $(\Delta, \Gamma)$ satisfies $\left(S_{r}\right)$ then $\mathbb{K}[\Delta, \Gamma]$ satisfies the assumptions of Lemma 4.2. Substituting the upper bounds of Lemma 4.2 into the definition of $\tilde{\sigma}$-numbers leads to the following upper bounds on their alternating sums. (We omit the computation as it is exactly the same as in the proof of [15, Proposition 6.2] but with $d+1$ replaced by $d$.)

Lemma 4.3. If a $(d-1)$-dimensional relative simplicial complex $(\Delta, \Gamma)$ satisfies Serre's condition $\left(S_{r}\right)$ over $\mathbb{K}$ then

$$
\sum_{j=0}^{i}(-1)^{i-j} \tilde{\sigma}_{j-1}(\Delta, \Gamma ; \mathbb{K}) \leq \frac{1}{d+1} \sum_{j=0}^{i}(-1)^{i-j} \frac{h_{j}(\Delta, \Gamma)}{\left(\begin{array}{l}
d \\
j
\end{array}\right)} \text { for } i \leq r-1 .
$$

We are now in a position to prove Theorem 1.4 for the class of $d$-dimensional pure relative simplicial complexes all of whose vertex links satisfy $\left(S_{r}\right)$. Recall that a pure relative simplicial complex $(\Delta, \Gamma)$ is called Buchsbaum (over $\mathbb{K}$ ) if for every vertex $v$ of $\Delta,(\operatorname{lk}(v, \Delta), \operatorname{lk}(v, \Gamma))$ is Cohen-Macaulay (over $\mathbb{K})$. In other words, a $d$-dimensional $(\Delta, \Gamma)$ is Buchsbaum if and only if it is pure and all vertex links of $(\Delta, \Gamma)$ satisfy $\left(S_{d}\right)$.

Proof of Theorem 1.4: Let $V$ be the vertex set of $\Delta$. Since $(\Delta, \Gamma)$ is pure, it follows from [21, Proposition 2.3] that

$$
i h_{i}(\Delta, \Gamma)+(d-i+2) h_{i-1}(\Delta, \Gamma)=\sum_{v \in V} h_{i-1}(\operatorname{lk}(v, \Delta), \operatorname{lk}(v, \Gamma))
$$

for $i=1,2, \ldots, d+1$. This result together with Lemmas 2.2 and 4.3 yields that for all $i \leq r$,

$$
\begin{aligned}
\sum_{j=1}^{i}(-1)^{i-j} \mu_{j-1}(\Delta, \Gamma ; \mathbb{K}) & =\sum_{j=1}^{i}(-1)^{i-j}\left(\sum_{v \in V} \tilde{\sigma}_{j-2}(\operatorname{lk}(v, \Delta), \operatorname{lk}(v, \Gamma) ; \mathbb{K})\right) \\
& \leq \sum_{j=1}^{i}(-1)^{i-j} \frac{1}{(d+1)\left(\begin{array}{c}
d \\
j-1
\end{array}\right)}\left(\sum_{v \in V} h_{j-1}(\operatorname{lk}(v, \Delta), \operatorname{lk}(v, \Gamma))\right) \\
& =\sum_{j=1}^{i}(-1)^{i-j} \frac{1}{(d+1)\left(\begin{array}{c}
d \\
j-1
\end{array}\right)}\left(j h_{j}(\Delta, \Gamma)+(d-j+2) h_{j-1}(\Delta, \Gamma)\right) \\
& =\sum_{j=1}^{i}(-1)^{i-j}\left(\frac{h_{j}(\Delta, \Gamma)}{\left(\begin{array}{c}
d+1 \\
j
\end{array}\right)}+\frac{h_{j-1}(\operatorname{lk}(v, \Delta), \operatorname{lk}(v, \Gamma))}{\left(\begin{array}{c}
d+1 \\
j-1
\end{array}\right)}\right) \\
& =\frac{h_{i}(\Delta, \Gamma)}{\left(\begin{array}{c}
d+1 \\
i
\end{array}\right)}-(-1)^{i} h_{0}(\Delta, \Gamma) .
\end{aligned}
$$

Since, by definition, $h_{0}(\Delta, \Gamma)=f_{-1}(\Delta, \Gamma)$, the above inequality completes the proof.

As we discussed in the Introduction, Theorem 1.3 is an immediate consequence of Inequality (1.3) and the $(r=2 \& \Gamma=\emptyset)$-case of Theorem [1.4.

We close this section with one additional remark. According to Theorem 1.4 , if $(\Delta, \Gamma)$ is a $d$-dimensional Buchsbaum relative simplicial complex, then

$$
h_{i}(\Delta, \Gamma) \geq\left(\begin{array}{c}
d+1 \\
i
\end{array}\right)\left(\sum_{j=1}^{i}(-1)^{i-j} \mu_{j-1}(\Delta, \Gamma ; \mathbb{K})+(-1)^{i} f_{-1}(\Delta, \Gamma)\right) \quad \text { for all } i \leq d .
$$


These inequalities provide a strengthening of a previously known fact [17, Theorem 3.4] that for a Buchsbaum $(\Delta, \Gamma), h_{i}(\Delta, \Gamma) \geq\left(\begin{array}{c}d+1 \\ i\end{array}\right) \sum_{j=1}^{i}(-1)^{i-j} \tilde{b}_{j-1}(\Delta, \Gamma ; \mathbb{K})$ for all $i \leq d$.

\section{Simplicial posets}

The goal of this section is to explain how the proofs of Theorems 1.3 and 1.4 can be extended to the generality of simplicial posets. This requires a quick review of the definition of simplicial posets and related notions as well as of the corresponding algebraic background.

A simplicial poset $\Delta=(\Delta, \preceq)$ is a finite poset with a unique minimal element, $\emptyset$, and with the property that for every $F \in \Delta$, the interval $[\emptyset, F]$ is a Boolean lattice [20]. Consequently, $\Delta$ is graded and atomic. Furthermore, it follows from the results of [5] that any simplicial poset $\Delta$ is the face poset of a certain regular CW-complex, $|\Delta|$, all of whose closed cells are simplices. Thus, one can think of a simplicial poset as a collection of simplices glued together in a way that every two simplices intersect along an arbitrary (possibly empty) subcomplex of their boundaries. Another important consequence of [5] is that $|\Delta|$ has a well-defined barycentric subdivision, denoted by $\operatorname{sd}(\Delta)$, which is the simplicial complex isomorphic to the order complex of $\Delta-\{\emptyset\}$ and homeomorphic to $|\Delta|$. In particular, the (singular) Betti numbers and the fundamental group of $|\Delta|$ coincide with the simplicial Betti numbers and the fundamental group of $\operatorname{sd}(\Delta)$, respectively.

From now on we refer to a simplicial poset $\Delta$ and its realization $|\Delta|$ almost interchangeably. The elements of $\Delta$ are called faces and the elements of rank 1 are called vertices. The dimension of a face $F$ is defined as the rank of the interval $[\emptyset, F]$ minus 1 , and the vertex set of $F$ is defined by $V(F)=\{v \in \Delta: \operatorname{rk}(v)=1, v \preceq F\}$.

Since all the faces of a simplicial poset are simplices, many definitions pertaining to simplicial complexes have natural extensions to simplicial posets. For instance, if $\Delta$ is a simplicial poset and $v$ is a vertex of $\Delta$, then the link of $v$ in $\Delta$ is defined by $\operatorname{lk}(v, \Delta)=\{F \in \Delta: v \preceq F\}$. It is easy to check that $\operatorname{lk}(v, \Delta)$ is a simplicial poset with minimal element $v$, and that $\operatorname{sd}(\operatorname{lk}(v, \Delta))$ is isomorphic to $\operatorname{lk}(v, \operatorname{sd}(\Delta)$ ). (However, it is worth pointing out that, in contrast to the setting of simplicial complexes, a vertex link of a simplicial poset $\Delta$ is not naturally a subcomplex of $\Delta$ : for instance, consider the link of a vertex in a simplicial poset consisting of two vertices and three edges joining them.) Similarly, for a subset $W$ of vertices of $\Delta$, the restriction of $\Delta$ to $W$ is defined by $\Delta_{W}=\{F \in \Delta: V(F) \subseteq W\}$.

In analogy with relative simplicial complexes, a relative simplicial poset is a pair of simplicial posets $(\Delta, \Gamma)$ such that $\Gamma \subseteq \Delta$ is a lower ideal of $\Delta$. The dimension of a relative simplicial poset $(\Delta, \Gamma)$ is $\operatorname{dim}(\Delta, \Gamma)=\max \{\operatorname{dim} F: F \in \Delta \backslash \Gamma\}$. As in the case of relative simplicial complexes, we denote by $f_{i}=f_{i}(\Delta, \Gamma)$ the number of $i$-dimensional faces of $\Delta \backslash \Gamma$ and by $\left(f_{-1}, f_{0} \ldots, f_{\operatorname{dim}(\Delta, \Gamma)}\right)$ the $f$-vector of $(\Delta, \Gamma)$. With the above notions in hand, the definitions from Section 2 of the $h$-vector, the $\mu^{\varsigma}$-numbers, and the $\mu$-numbers for a relative simplicial complex carry over verbatim to the definitions of the same objects for a relative simplicial poset.

The following lemma provides a connection between the $\mu$-numbers of a relative simplicial poset $(\Delta, \Gamma)$ and the $\mu$-numbers of its barycentric subdivision - the relative simplicial complex $(\operatorname{sd}(\Delta), \operatorname{sd}(\Gamma))$. If $\Delta$ is a simplicial poset with vertex set $V,|V|=n$, and $\varsigma=\left(v_{1}, \ldots, v_{n}\right) \in \mathcal{S}(V)$, we define an extension of $\varsigma$ to an ordering $\operatorname{sd}(\varsigma)$ of the vertices of $\operatorname{sd}(\Delta)$ by inserting between $v_{k-1}$ and $v_{k}$ the barycenters of the faces of $\Delta_{\left\{v_{1}, \ldots, v_{k}\right\}}$ that contain $v_{k}$, listing them in the increasing order of the dimension of their faces. (Note that the choice of $\operatorname{sd}(\varsigma)$ may not be unique.) 
Lemma 5.1. Let $(\Delta, \Gamma)$ be a relative simplicial poset, let $V$ be the vertex set of $\Delta$, and let $\varsigma \in \mathcal{S}(V)$. For an arbitrary field $\mathbb{K}$ and for all $i \geq 0$,

$$
\mu_{i}^{\varsigma}(\Delta, \Gamma ; \mathbb{K})=\mu_{i}^{\operatorname{sd}(\varsigma)}(\operatorname{sd}(\Delta), \operatorname{sd}(\Gamma) ; \mathbb{K}) .
$$

In particular, the $\mu^{\varsigma}$-numbers of $(\Delta, \Gamma)$, and hence also the $\mu$-numbers of $(\Delta, \Gamma)$, satisfy the Morse inequalities of Lemma 2.1; furthermore, if $\Delta$ is connected, then $\mu_{1}(\Delta)-\mu_{0}(\Delta)+1 \geq m(\Delta)$.

Proof: $\quad$ Let $U$ be the vertex set of $\operatorname{sd}(\Delta)$. When $w \in U$, we denote by $\operatorname{sd}(\Delta)_{\leq w}$ and $\operatorname{sd}(\Gamma)_{\leq w}$ the subcomplexes of $\operatorname{sd}(\Delta)$ and $\operatorname{sd}(\Gamma)$, respectively, induced by $\{u \in U: u \leq w\}$ - the initial segment of $U$ in the ordering $\operatorname{sd}(\varsigma)$. As follows from (2.1), to prove (5.1), it suffices to show that for each vertex $v_{k} \in V$, the contribution of $v_{k}$ to both sides of (5.1) is the same, that is,

$$
\tilde{b}_{i-1}\left(\operatorname{lk}\left(v_{k}, \Delta_{\left\{v_{1}, \ldots, v_{k}\right\}}\right), \operatorname{lk}\left(v_{k}, \Gamma_{\left\{v_{1}, \ldots, v_{k}\right\}}\right) ; \mathbb{K}\right)=\tilde{b}_{i-1}\left(\operatorname{lk}\left(v_{k}, \operatorname{sd}(\Delta)_{\leq v_{k}}\right), \operatorname{lk}\left(v_{k}, \operatorname{sd}(\Gamma)_{\leq v_{k}}\right) ; \mathbb{K}\right),
$$

while for each $w \in U \backslash V$, the contribution of $w$ to the right-hand side of (5.1) is zero, that is,

$$
\tilde{b}_{i-1}\left(\operatorname{lk}\left(w, \operatorname{sd}(\Delta)_{\leq w}\right), \operatorname{lk}\left(w, \operatorname{sd}(\Gamma)_{\leq w}\right) ; \mathbb{K}\right)=0 .
$$

Indeed, the former equation holds since the relative complex $\left(\operatorname{lk}\left(v_{k}, \operatorname{sd}(\Delta)_{\leq v_{k}}\right), \operatorname{lk}\left(v_{k}, \operatorname{sd}(\Gamma)_{\leq v_{k}}\right)\right)$ is the barycentric subdivision of $\left(\operatorname{lk}\left(v_{k}, \Delta_{\left\{v_{1}, \ldots, v_{k}\right\}}\right), \operatorname{lk}\left(v_{k}, \Gamma_{\left\{v_{1}, \ldots, v_{k}\right\}}\right)\right)$. As for the latter equation, suppose that $w$ is the barycenter of a face $F$ of $\Delta$, and that $v_{k}$ is the maximal vertex of $F$ in the ordering $\varsigma$. If $F$ is not a face of $\Gamma$, then $\operatorname{lk}\left(w, \operatorname{sd}(\Gamma)_{\leq w}\right)=\emptyset$ while $\operatorname{lk}\left(w, \operatorname{sd}(\Delta)_{\leq w}\right)$ is the complex obtained by barycentrically subdividing the boundary of the simplex $F$ and then deleting the vertex $v_{k}$. Thus, in this case $\left(\operatorname{lk}\left(w, \operatorname{sd}(\Delta)_{\leq w}\right), \operatorname{lk}\left(w, \operatorname{sd}(\Gamma)_{\leq w}\right)\right)=\operatorname{lk}\left(w, \operatorname{sd}(\Delta)_{\leq w}\right)$ is contractible. On the other hand, if $F$ is also a face of $\Gamma$, then so are all the faces of $F$. Hence $\operatorname{lk}\left(w, \operatorname{sd}(\Delta)_{\leq w}\right)=\operatorname{lk}\left(w, \operatorname{sd}(\Gamma)_{\leq w}\right)$, that is, $\left(\operatorname{lk}\left(w, \operatorname{sd}(\Delta)_{\leq w}\right), \operatorname{lk}\left(w, \operatorname{sd}(\Gamma)_{\leq w}\right)\right)=\emptyset$. In either case, (5.2) follows.

We now turn our discussion to analogs of Stanley-Reisner rings and modules (introduced in [20]) for simplicial posets. Let $\Delta$ be a simplicial poset. Consider the polynomial ring $\tilde{S}=\mathbb{K}\left[x_{F}\right.$ : $F \in \Delta]$ with one variable per each face of $\Delta$. The face ideal of $\Delta, J_{\Delta}$, is the ideal of $\tilde{S}$ generated by the elements of the following form:

- $x_{F} x_{G}$ for all pairs of elements $F, G \in \Delta$ that have no common upper bound in $\Delta$;

- $x_{F} x_{G}-x_{F \wedge G} \sum x_{H}$ for pairs of $F, G$ incomparable in $\Delta$, where the sum is over the set of all minimal upper bounds of $F$ and $G$. (If $F$ and $G$ have an upper bound $H$, then $F$ and $G$ are elements of $[\emptyset, H]$, which is a Boolean lattice, and so $F \wedge G$ is well defined.)

The ring $\mathbb{K}[\Delta]=\tilde{S} / J_{\Delta}$ is called the face ring of $\Delta$. If $(\Delta, \Gamma)$ a simplicial poset, then we define the face module of $(\Delta, \Gamma)$ as $\mathbb{K}[\Delta, \Gamma]=J_{\Gamma} / J_{\Delta}$.

The key that allows us to extend Theorems 1.3 and 1.4 to the generality of simplicial posets comes from the body of work showing that the face rings and modules of simplicial posets enjoy many of the same properties that the Stanley-Reisner rings and modules of simplicial complexes do. For instance, if $\Delta$ is a simplicial poset of dimension $d-1$, then (1) the Krull dimension of $\mathbb{K}[\Delta]$ is $d$ and the $\mathbb{Z}$-graded Hilbert series of $\mathbb{K}[\Delta]$ is given by $(1-x)^{-d} \sum_{i=0}^{d} h_{i}(\Delta) x^{i}$ (see [20, Proposition 3.8]); (2) $\mathbb{K}[\Delta]$ is a finitely generated graded module over $S=\mathbb{K}\left[x_{v}: v \in \Delta, \operatorname{rk}(v)=1\right]$ 
and the Betti numbers of $\mathbb{K}[\Delta], \beta_{i, j}(\mathbb{K}[\Delta])=\operatorname{dim}_{\mathbb{K}} \operatorname{Tor}_{i}^{S}(\mathbb{K}[\Delta], \mathbb{K})_{j}$, satisfy Hochster's formula that expresses these numbers in terms of simplicial Betti numbers of induced subposets of $\Delta$ (see [9, Corollary 4.6]). In fact, it is not hard to see that Duval's proof of this latter fact can be generalized to the case of $\mathbb{K}[\Delta, \Gamma]$, where $(\Delta, \Gamma)$ is a relative simplicial poset. Consequently, Lemma 2.2 continues to hold for relative simplicial posets. Finally, since face modules of simplicial posets are squarefree modules (this follows from [23, Lemma 2.3] and [24, Lemma 2.5]) and since the proof of Theorem 4.1 applies to all squarefree modules, we conclude that Theorem 4.1 holds in the generality of face modules of simplicial posets.

Therefore, all results of Section 4, including Theorem 1.4, continue to hold for relative simplicial posets. Furthermore, the last part of Lemma 5.1 combined with Theorem 1.4 implies that Theorem 1.3 also continues to hold for simplicial posets. In fact, the inequalities of Theorem 1.3 are sharp for simplicial posets; this follows from [7, Theorem 2.5] and the Morse inequalities.

\section{References}

[1] Karim A. Adiprasito and Raman Sanyal. Relative Stanley-Rreisner theory and upper bound theorems for Minkowski sums. Publ. Math. Inst. Hautes Études Sci., to appear; http://link.springer.com/article/10.1007/s10240-016-0083-7, 2016.

[2] Christos A. Athanasiadis and Volkmar Welker. Buchsbaum* complexes. Math. Z., 272(1-2):131-149, 2012.

[3] Bhaskar Bagchi and Basudeb Datta. On stellated spheres and a tightness criterion for combinatorial manifolds. European J. Combin., 36:294-313, 2014.

[4] David Barnette. A proof of the lower bound conjecture for convex polytopes. Pacific J. Math., 46:349-354, 1973.

[5] A. Björner. Posets, regular CW complexes and Bruhat order. European J. Combin., 5(1):7-16, 1984.

[6] U. Brehm and W. Kühnel. Combinatorial manifolds with few vertices. Topology, 26(4):465-473, 1987.

[7] Jonathan Browder and Steven Klee. A classification of the face numbers of Buchsbaum simplicial posets. Math. Z., 277(3-4):937-952, 2014.

[8] Winfried Bruns and Jürgen Herzog. Cohen-Macaulay rings, volume 39 of Cambridge Studies in Advanced Mathematics. Cambridge University Press, Cambridge, 1996. Revised edition.

[9] Art M. Duval. Free resolutions of simplicial posets. J. Algebra, 188(1):363-399, 1997.

[10] Allen L. Fogelsanger. The generic rigidity of minimal cycles. ProQuest LLC, Ann Arbor, MI, 1988. Thesis (Ph.D.)-Cornell University.

[11] Gil Kalai. Rigidity and the lower bound theorem. I. Invent. Math., 88(1):125-151, 1987.

[12] Steven Klee. The fundamental group of balanced simplicial complexes and posets. Electron. J. Combin., 16(2, Special volume in honor of Anders Björner):Research Paper 7, 12, 2009.

[13] Satoshi Murai. Tight combinatorial manifolds and graded Betti numbers. Collect. Math., 66(3):367$386,2015$.

[14] Satoshi Murai and Eran Nevo. On $r$-stacked triangulated manifolds. J. Algebraic Combin., 39(2):373388, 2014.

[15] Satoshi Murai and Isabella Novik. Face numbers of manifolds with boundary. Int. Math. Res. Not., to appear, 2016. 
[16] Satoshi Murai and Naoki Terai. $h$-vectors of simplicial complexes with Serre's conditions. Math. Res. Lett., 16(6):1015-1028, 2009.

[17] Isabella Novik and Ed Swartz. Socles of Buchsbaum modules, complexes and posets. Adv. Math., 222(6):2059-2084, 2009.

[18] Isabella Novik and Ed Swartz. Face numbers of pseudomanifolds with isolated singularities. Math. Scand., 110(2):198-222, 2012.

[19] Peter Schenzel. Dualisierende Komplexe in der lokalen Algebra und Buchsbaum-Ringe, volume 907 of Lecture Notes in Mathematics. Springer-Verlag, Berlin-New York, 1982.

[20] Richard P. Stanley. f-vectors and h-vectors of simplicial posets. J. Pure Appl. Algebra, 71(2-3):319$331,1991$.

[21] Ed Swartz. Lower bounds for $h$-vectors of $k$-CM, independence and broken circuit complexes. SIAM J. Disc. Math., 18(3):647-661, 2004/05.

[22] Ed Swartz. Face enumeration-from spheres to manifolds. J. Eur. Math. Soc. (JEMS), 11(3):449-485, 2009.

[23] Kohji Yanagawa. Alexander duality for Stanley-Reisner rings and squarefree $\mathbf{N}^{n}$-graded modules. $J$. Algebra, 225(2):630-645, 2000.

[24] Kohji Yanagawa. Dualizing complex of the face ring of a simplicial poset. J. Pure Appl. Algebra, 215(9):2231-2241, 2011. 